University of Nebraska - Lincoln

DigitalCommons@University of Nebraska - Lincoln

\title{
Role of helmet in the mechanics of shock wave propagation under blast loading conditions
}

\author{
Shailesh G. Ganpule \\ University of Nebraska-Lincoln \\ Linxia Gu \\ University of Nebraska-Lincoln, gul@fit.edu \\ Aaron L. Alai \\ University of Nebraska-Lincoln, aalai2@unl.edu \\ Namas Chandra \\ University of Nebraska-Lincoln, nchandra2@unl.edu
}

Follow this and additional works at: https://digitalcommons.unl.edu/mechengfacpub

Part of the Mechanical Engineering Commons

Ganpule, Shailesh G.; Gu, Linxia; Alai, Aaron L.; and Chandra, Namas, "Role of helmet in the mechanics of shock wave propagation under blast loading conditions" (2011). Mechanical \& Materials Engineering Faculty Publications. 56.

https://digitalcommons.unl.edu/mechengfacpub/56

This Article is brought to you for free and open access by the Mechanical \& Materials Engineering, Department of at DigitalCommons@University of Nebraska - Lincoln. It has been accepted for inclusion in Mechanical \& Materials Engineering Faculty Publications by an authorized administrator of DigitalCommons@University of Nebraska Lincoln. 
Published in Computer Methods in Biomechanics and Biomedical Engineering (2011), doi: 10.1080/10255842.2011.597353.

Copyright $(\odot 2011$ Taylor \& Francis. Used by permission. http://www.tandf.co.uk/journals/gcmb

Submitted December 24, 2010; revised and accepted June 13, 2011.

\title{
Role of helmet in the mechanics of shock wave propagation under blast loading conditions
}

\author{
S. Ganpule, ${ }^{1}$ L. Gu, ${ }^{2}$ A. Alai, ${ }^{1}$ and N. Chandra ${ }^{1}$ \\ 1. Department of Engineering Mechanics, University of Nebraska-Lincoln, Lincoln, NE 68588-0526, USA \\ 2. Department of Mechanical Engineering, University of Nebraska-Lincoln, Lincoln, NE 68588-0656, USA \\ Corresponding author - N. Chandra, email nchandra2@unl.edu
}

\begin{abstract}
The effectiveness of helmets in extenuating the primary shock waves generated by the explosions of improvised explosive devices is not clearly understood. In this work, the role of helmet on the overpressurization and impulse experienced by the head were examined. The shock wave-head interactions were studied under three different cases: (i) unprotected head, (ii) head with helmet but with varying head-helmet gaps and (iii) head covered with helmet and tightly fitting foam pads. The intensification effect was discussed by examining the shock wave flow pattern and verified with experiments. A helmet with a better protection against shock wave is suggested.
\end{abstract}

Keywords: blast, head, helmet, TBI, FSI

\section{Introduction}

Improvised explosive device explosions with increasing strengths and sophistication, in the current military conflicts, result in blast traumatic brain injuries (bTBI) to the soldiers wearing tightly/loosely fitting helmets, or to the civilians even without helmets. Defense and Veterans Brain Injury Center has reported that there are 188,270 clinically confirmed TBI cases within the US service members from 2000 to the second quarter of 2010. In the case of mild TBI, there are currently no medical diagnostic tools or biomarkers that indicate the onset of the ailment, further endangering the battle crew to further exposures. It is speculated that bTBI is a stress wave dominated phenomenon as opposed to rotational acceleration/deceleration-induced injury, typically associated with the impact TBI encountered in sports and automobile accidents (Cernak et al. 2001; Courtney and Courtney 2009; Moore et al. 2009; Moss et al. 2009; Taylor and Ford 2009). However, our current understanding of bTBI remains incomplete (Ling et al. 2009). The knowledge of detailed mechanisms of blast waves-head interactions is the key in developing effective strategy to reduce the occurrence of TBI.

Current military helmets provide considerable protection against penetrating ballistic injury, whereas their protection against the blast overpressure is not clear because they are neither designed for nor tested under blast loading conditions (Lew et al. 2005; Okie 2005; Xydakis et al. 2007; Bhattacharjee 2008). A few researchers (Mott et al. 2008; Moss et al. 2009) have conducted preliminary investigations on the performance of the helmet under blast loadings and concluded that helmets tend to enhance local overpressure on the head by focused interactions of the blast waves. However, they do not provide critical understanding of this phenomenon to substantiate their claim; in addition, they do not show how this focusing affects the load transmitted to the head.

The main objective of this work was to understand the physics of flow past the human head with and without helmets, subjected to primary shockwave loading conditions. A secondary but equally important objective is to understand the role of geometry of the head-helmet subspace (e.g. gaps, pads and curvature) on the mechanics of flow and hence the loading on the head. In Section 2, we describe finite element (FE) discretization, material models, boundary conditions and the method of solution. In Section 3, numerical results are presented and discussed and compared with the experiments. Specific observations on the effect of helmets on the loading experienced by the head are presented in Section 4.

\section{Methods}

\subsection{FE discretization}

The head and the helmet are modeled with Lagrangian elements, and the surrounding fluid medium, in which shock wave propagates, is modeled with Eulerian elements (Figure 1). The size of the Eulerian domain is selected such that the reflections from domain boundaries are negligible and the shock wave profile is purely 1D. The head model is generated from the segmentation of high-resolution MRI data $(192 \mathrm{~mm} \times 256 \mathrm{~mm} \times 256 \mathrm{~mm})$ obtained from the Visible Human Project. The head is segmented into the skull and the brain (Figure 1). Brain and cerebrospinal fluid are not segmented separately and membranes are not included. The interface between the skull and the brain is 


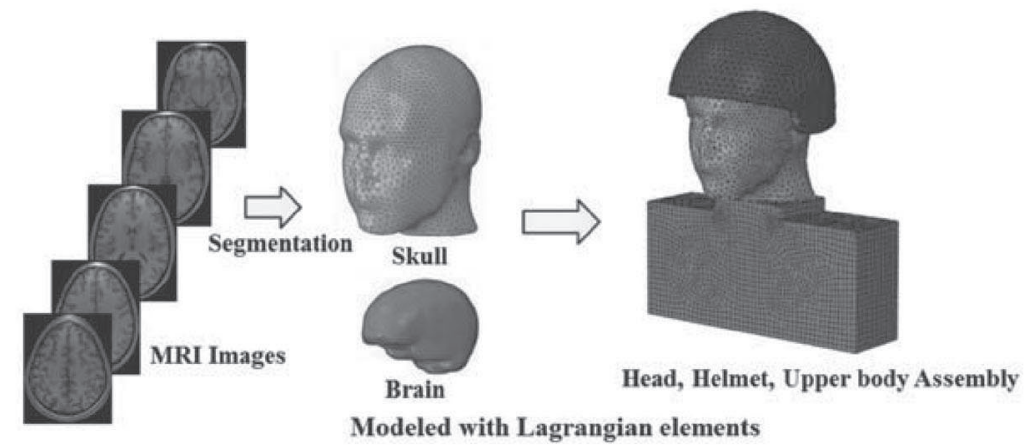

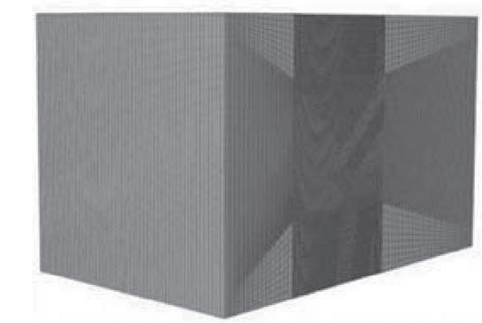

Surrounding medium (Modeled with Eulerian elements)

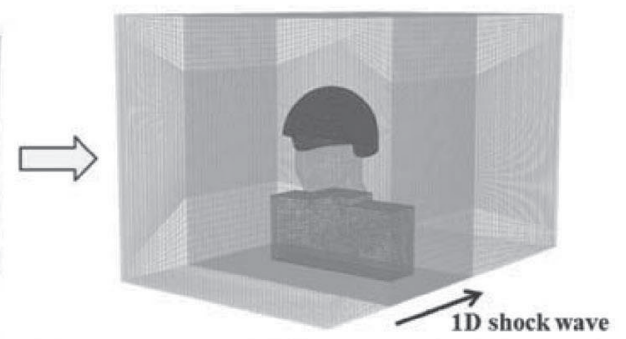

Lagrangian bodies immersed in Eulerian Domain

Figure 1. FE discretization.

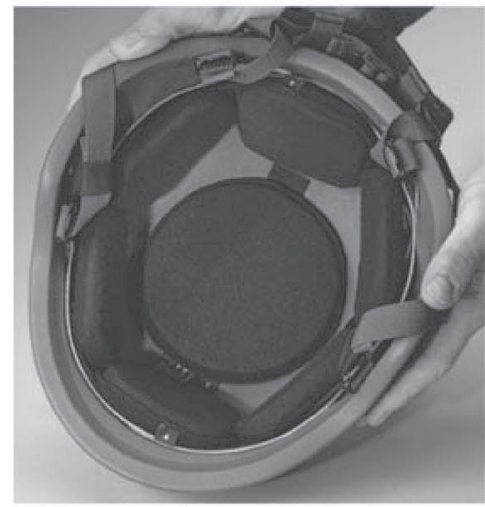

Seven pad suspension system

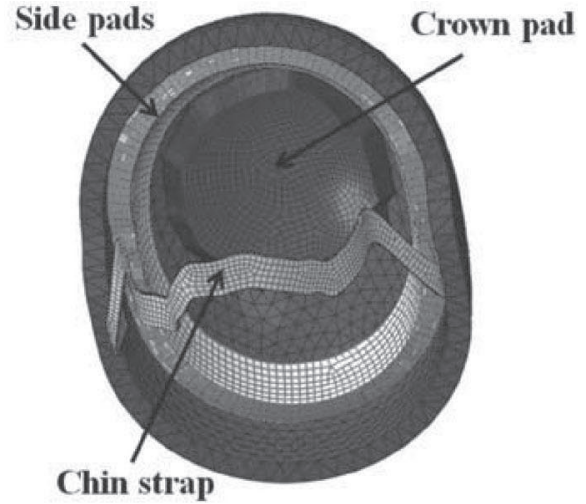

FE approximation of suspension system

Figure 2. Pads suspension system.

modeled through penalty contact algorithm with tangential sliding and hard contact normal behavior. The model of the helmet is generated by digitizing advanced combat helmet $(\mathrm{ACH})$. These geometric models are discretized in the HyperMesh ${ }^{\circledR}$ to yield 111,204 and 6,719 tetrahedral elements on the head and the helmet, respectively. Eulerian domain consists of 1,044,948 brick elements with appropriate mesh refinement near the regions of solid bodies to capture fluid-structure interaction (FSI) effects. The head and the helmet are assembled together with an offset of $\sim 13 \mathrm{~mm}(\mathrm{~g}=13 \mathrm{~mm})$ from the skull as per ballistic standard (Reynosa 1999). In the case of foam padding between the helmet and the head, the head is partially connected to the helmet through seven-pad suspension system shown in Figure 2. For capturing the possible effects of upper body reflections, shoulders and upper body are added (Figure 1).
This head, helmet, upper body assembly is immersed into Eulerian domain that essentially models the surrounding atmosphere in which shock wave propagates. The 3D simulations were computationally exhaustive and carried out for studying three base cases: without helmet, with helmet and gap, and with helmet and pads. For carrying out parametric studies on the geometry and the loadings, 2D simulations were carried out. In these cases, the head is simplified as a circular cylinder and the helmet as a semicircular cylinder with a constant offset from the head.

\subsection{Material models}

The skull is modeled as linear, elastic, isotropic material with properties adopted from the literature, the details 
of which can be found elsewhere (Ganpule et al. 2010). Elastic properties, in general, are sufficient to capture the wave propagation characteristics and are consistent with other research works (Moore et al. 2009; Moss et al. 2009; Chafi et al. 2010; Chen and Ostoja-Starzewski 2010). The brain volumetric response is characterized as linear, elastic and isotropic, whereas the brain shear response is characterized as linear, isotropic, and viscoelastic and the properties are adopted from Zhang et al. (2001). The Kevlar helmet is modeled as transversely isotropic elastic material with properties obtained from Aare and Kleiven (2007). The foam pads are modeled with a linear bulk response and a viscoelastic shear response. The properties of foam pads are taken from Moss et al. (2009), who obtained the properties from low-rate compression and acoustic testing of $\mathrm{ACH}$ foam pads. The air is modeled as an ideal gas equation given by

$$
P=(\gamma-1) \frac{\rho}{\rho_{0}} e
$$

where $P$ is the pressure, $\gamma$ is the constant pressure to constant volume specific heat ratio (1.4 for air), $\rho_{0}$ is the initial air mass density, and $\rho$ is the current mass density and $e$ is the internal volumetric energy density. The shock wave Mach number in our simulations is $\sim 2$. Hence, ideal gas equation of state assumption is valid, as ratio of specific heats do not change drastically for this Mach number. The material properties are listed in Table 1.

\subsection{Loading and boundary conditions}

Loading and boundary conditions are shown in Figure 3 . The shock wave is created by releasing high-pressure air into atmospheric air at time $t=0$. The density of highpressure air is adjusted so as to produce desired incident blast overpressures near the head. Frontal blast loading scenario is simulated. As the shock wave propagates through the Eulerian fluid domain, it applies dynamic loading on the Lagrangian solid domain. Blast peak overpressures are selected based on blast injury thresholds proposed by Courtney and Courtney (2011) and Bowen et al. (1968). The following three blast overpressure conditions are used:

(a) Blast peak overpressure of $0.18 \mathrm{MPa}$ corresponds to positive pulse duration of $0.65 \mathrm{~ms}$. This value is based on the injury threshold proposed by Courtney and Courtney (2011).

(b) Blast peak overpressure of $0.52 \mathrm{MPa}$ corresponding to a blast-induced lung-injury threshold proposed by Bowen et al. (1968). It is to be noted that this peak overpressure is almost triple the peak overpressure in case (a) and is used only in 2D helmet head simulations carried out to understand the flow physics within headhelmet subspace.

(c) Average of cases (a) and (b) which corresponds to a peak overpressure of $0.35 \mathrm{MPa}$ to examine the trend between these overpressures.
The velocity perpendicular to each face of Eulerian domain is kept zero to avoid escaping/leaking of air through these faces (Figure 3(a)). This will create a pure 1D shock front traveling in the longitudinal direction with no lateral flow. The bottom face of the upper body is constrained in all six degrees of freedom to avoid rigid body translation (Figure 3(b)). The tied constraint is used between neck-upper body and helmet-chin strap. The interactions between a Eulerian region (containing air and a propagating blast wave) and a Lagrangian region are treated as an FSI. Contact pairs are defined through penalty contact algorithm with frictionless tangential sliding and hard contact normal behavior.

\subsection{Solution scheme}

This FE model is solved using nonlinear transient dynamic procedure with arbitrary Euler-Lagrangian coupling method (Abaqus ${ }^{\circledR}$, Providence, Rhode Island, USA). In this procedure, the governing partial differential equations for the conservation of momentum, mass and energy along with the material constitutive equations and the equations defining the initial and the boundary conditions are solved simultaneously. Eulerian framework allows modeling of highly dynamic events (e.g. shock), which will otherwise induce heavy-mesh distortion. In Abaqus ${ }^{\circledR}$, the Eulerian time incrementation algorithm is based on an operator split of the governing equations, resulting in a traditional Lagrangian phase followed by an Eulerian, or transport, phase. This formulation is known as "Lagrange plus remap." During the Lagrangian phase of the time, increment nodes are assumed to be temporarily fixed within the material, and elements deform with the material. During the Eulerian phase of the time, increment deformation is suspended, elements with significant deformation are automatically remeshed and the corresponding material flow between neighboring elements is computed. As material flows through a Eulerian mesh, state variables are transferred between elements by advection. Second-order advection is used in the current analysis. The Eulerian and Lagrangian elements use the same underlying formulation (including interpolation) with extensions to allow multiple materials and to support the Eulerian transport phase for Eulerian elements. In the current analysis, eight-node brick elements are used for Eulerian elements and 10-node tetrahedron for Lagrangian elements. These elements use isoparametric interpolation functions.

An enhanced immersed boundary method is used to provide the coupling between the Eulerian and the Lagrangian domains. Here, the Lagrangian region resides fully or partially within the Eulerian region and provides no-flow boundary conditions to the fluid in the direction normal to the local surface. Further, the Eulerian region provides the pressure boundary conditions to the Lagrangian region. Thus, combination of fixed Eulerian mesh and solid-fluid interface modeling through enhanced immersed boundary method allows for the concurrent simu- 


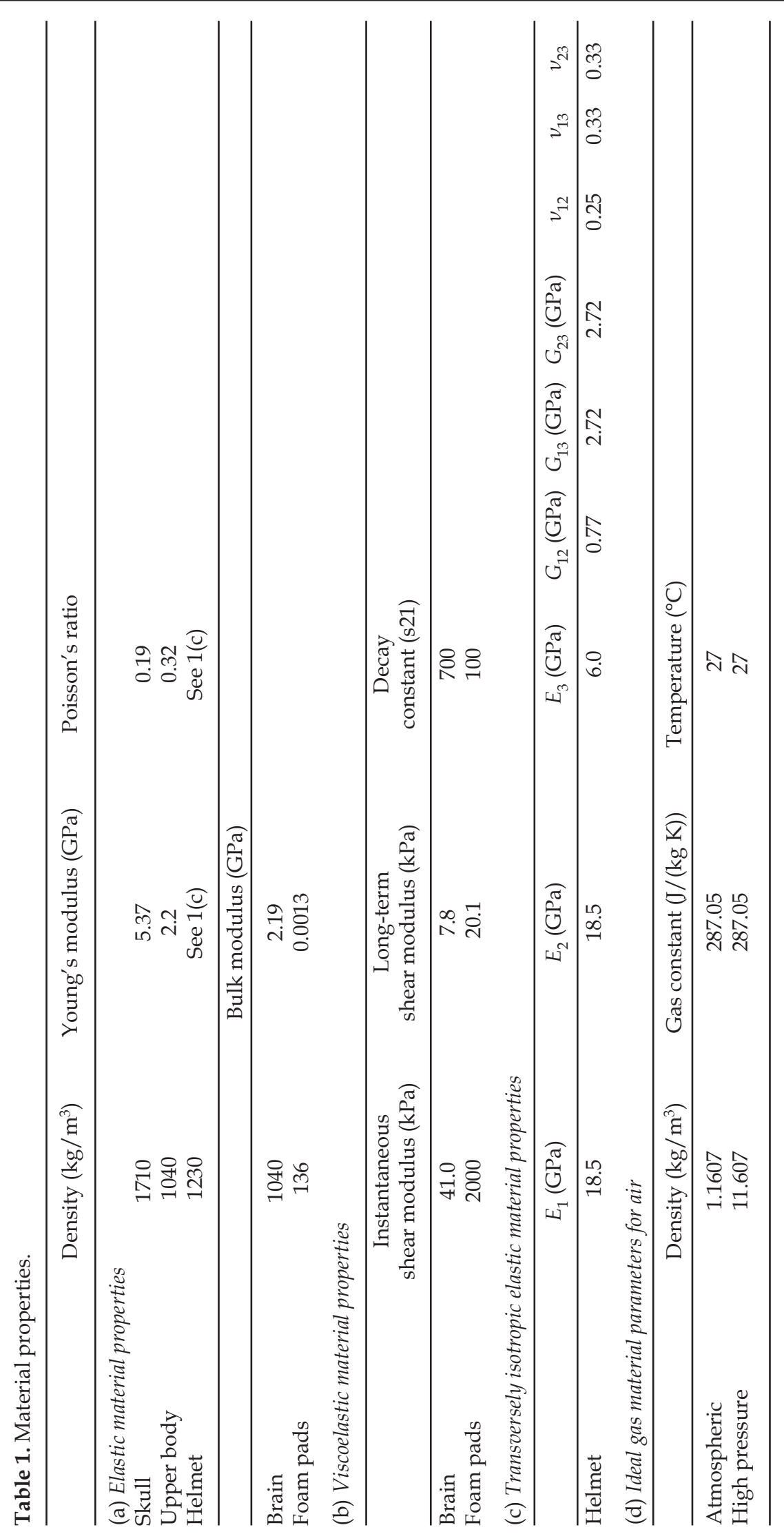




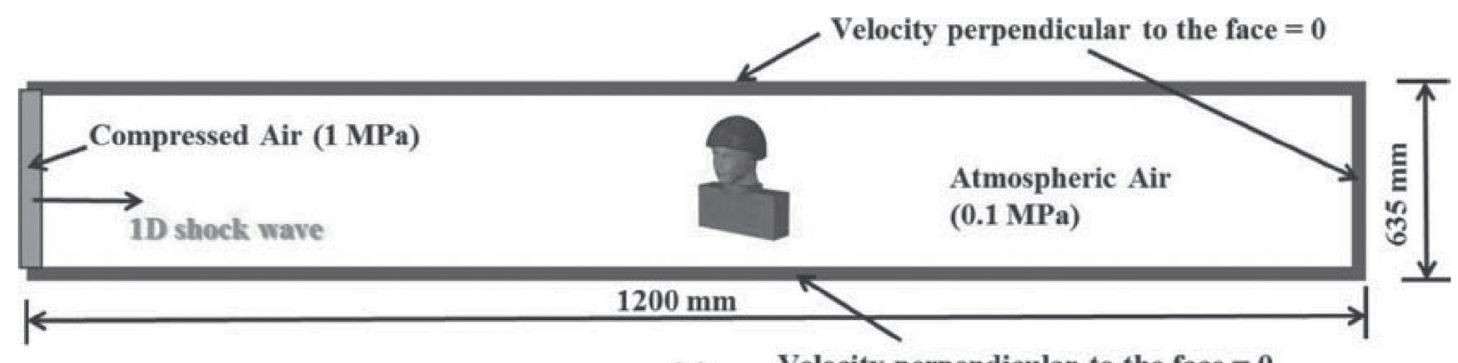

(a) Velocity perpendicular to the face $=0$

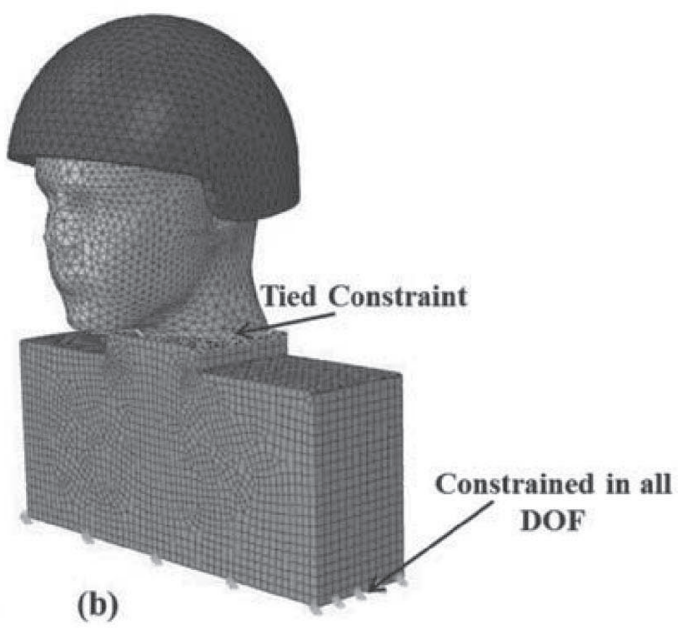

Figure 3. Loading and boundary conditions.

lations of the formation and propagation of primary shock wave in a fluid medium and accounts for the FSI effects and structural deformations once the shock wave encounters a solid. A typical 3D simulation requires about $14 \mathrm{~h}$ of CPU time, run on a dedicated 64 Opteron parallel processors (processor speed $2.2 \mathrm{GHz}, 2 \mathrm{~GB}$ memory per processor) for an integration time of $2 \mathrm{~ms}$. 2D simulations require about $1 \mathrm{~h}$ of CPU time using 64 Opteron parallel processors to achieve the physical event time of $4 \mathrm{~ms}$. Time step of the order of $1 \times 10^{27} \mathrm{~s}$ is essential to resolve and capture wave disturbances of the order of $1 \mathrm{MHz}$, and this increases the overall computational effort for the total simulation time of interest. These simulation times are selected based on the fact that even after the early time wave actions subside the peaks due to internal reflections continue to be established and the simulation needs to capture all these relevant events (Taylor and Ford 2009).

\section{Results and discussions}

First, we will demonstrate that the computational methodology is capable of generating 1D planar shockwave without any reflections from Eulerian domain boundaries. This is very important from both theoretical and experimental perspectives in that the effect of various phases of the wave (overpressure, underpressure, rise and total times) on material and structures can be clearly delineated. Next, the FSI at fluid-solid (head/helmet) interface is presented. The ef- fect of helmet on the flow field near the head is then presented. Effects of curvature of the helmet and the head, gap size between the helmet and the head and intensity of peak overpressure $P^{*}$ are then examined. Finally, the role of foam padding is examined and experimental results validating numerical trends are presented.

\subsection{Controlled 1D shock wave}

Figure 4 shows the pressure history at various locations before the blast wave impacts the head (with or without

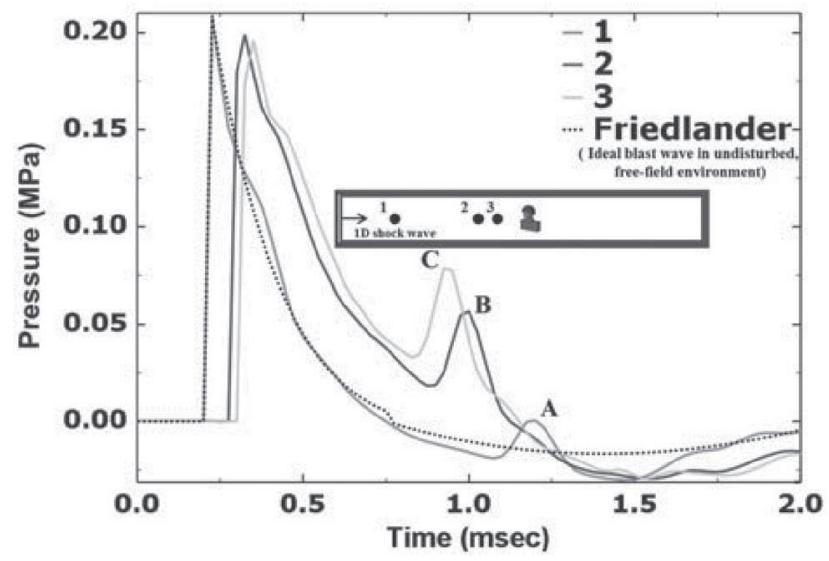

Figure 4. Pressure histories at various locations of the Eulerian domain as shock wave propagates through surrounding medium (3D simulations). 
helmet). Locations along the path of wave propagation are denoted as 1,2 , and 3 . The pressure-time history at these locations corresponds to a typical Friedlander 1D shock wave (Baker 1973). Many mathematical forms have been suggested to capture the time variation of ideal blast waves. Friedlander waveform represents one such form, which represents pressure-time history of an air blast in an undisturbed, free-field environment. The Friedlander waveform is given by Baker (1973)

$$
p(t)=p_{0}+P_{\mathrm{s}}^{+}\left(1-\frac{t}{T^{+}}\right) e^{-b t / T+}
$$

where $p$ denotes pressure; $t$, time; $p_{0^{\prime}}$ ambient pressure; $P_{s^{\prime}}^{+}$ peak overpressure or peak side on overpressure; $T^{+}$, positive phase duration and $b$, decay constant.

The Friedlander waveform occurs in open-field blasts in which no obstructions are present to generate complex wave reflections. Both the sharp rise and the exponential decay at these locations from our model closely follow Friedlander analysis (shown in dotted line). Thus, it can be concluded that the shock wave in our model is $1 \mathrm{D}$ planar. If there is any other wave disturbance, the pressure variation will not be smooth but oscillate. The peak overpressure of this shock wave is $0.18 \mathrm{MPa}$ in the vicinity of the head before it hits the head. The shock wave velocity can be determined from the arrival time and the distance of separation between two measurement stations and is estimated to be about $721 \mathrm{~m} / \mathrm{s}$ (Mach number $=2.10$ ). A set of second peaks (denoted as $\left.1^{\prime}, 2^{\prime}, 3^{\prime}\right)$ at locations 1, 2 and 3 corresponds to reflected waves from the head. The intensity of reflected pressure is maximum near the solid body and decreases as we move away from the head.

\subsection{Fluid-structure interaction}

When a shock wave encounters a solid surface, the incoming shock wave pressure is amplified due to FSI. The amplification factor depends on the incident shock strength, the fluid medium in which shock wave travels, angle of incidence, geometry, elastic and inertial properties of the target, and can vary from 2 to 8 (Anderson 2001). The pressure distribution in the vicinity of helmet-head (midsagittal section) and the pressure history at fluid-head interface are shown in Figure 5. As the blast wave approaches the body, it first encounters the helmet and is partially reflected back into the oncoming wave and partially deflected around the contour of the body. The reflected wave front has a maximum peak overpressure of 4.61 times the incident pressure. This factor is different at different parts of the face depending upon their shape. The maximum peak overpressure is observed near the nasion (corner of the eye socket and the nose wall), as shown in Figure 5.A number of numerical simulations (details not shown for brevity) clearly show that amplification factor is significantly higher for a concave geometry (compared with convex or flat), and further this factor depends on the radius of concavity and incident peak pressure. However, no perceptible difference is seen

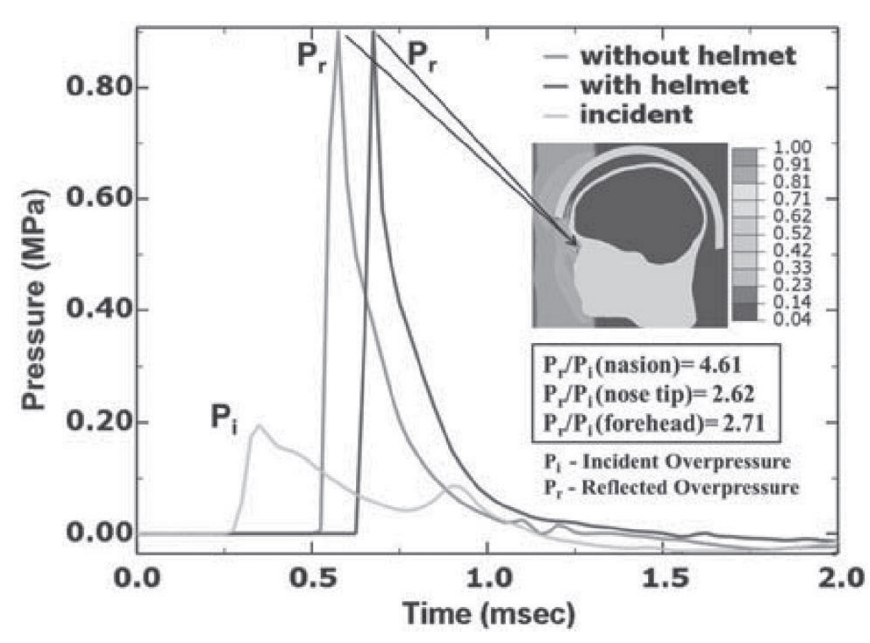

Figure 5. Pressure intensification at fluid-solid interface (3D simulations).

between flat and convex geometries. It is plausible that a concave surface reflects oncoming waves towards each other mutually reinforcing each other leading to a higher overpressure. These reinforcing waves explain why the nasion, which is concave, experiences the highest amplification factor. It should be noted that maximum overpressure is the same in both with and without helmet cases because the region of maximum reflected overpressure (nasion) is not covered by the helmet.

\subsection{Underwash effect of the helmet}

Figure 6 shows how the flow field evolves inside and outside of the head-helmet subspace. The blast front after encountering the head-helmet assembly is divided into two fronts: one front traveling around the outer perimeter of the helmet; another front penetrates the gap between the head and the helmet and travels underneath the helmet towards the back of the head as shown in Figure 6(a). The shock front traveling outside the helmet reaches the rear of the helmet before the shock front traverses through the gap (Figure 6(b-i)); and eventually when these two blast fronts meet, they focus on a region on the back side of the head (Figure 6(b-ii)). This process has been termed as underwash effect of the helmet (Moss et al. 2009). This underwash produces the higher peak pressures on the head, away from the direction of the incident wave when the location is shielded by the helmet. After this high pressure is generated, the high-pressure air in the head-helmet subspace expands in all the directions (Figure 6(b-iii)).

Figure 7 compares the pressure history at various locations of head-helmet subspace (midsagittal plane) of three base cases: without helmet, with helmet and gap, and with helmet and pads (to be discussed later). For the sake of convenience, let us call these three cases as 1, 2 and 3, respectively. When the helmet is present with a gap (case 2), the 


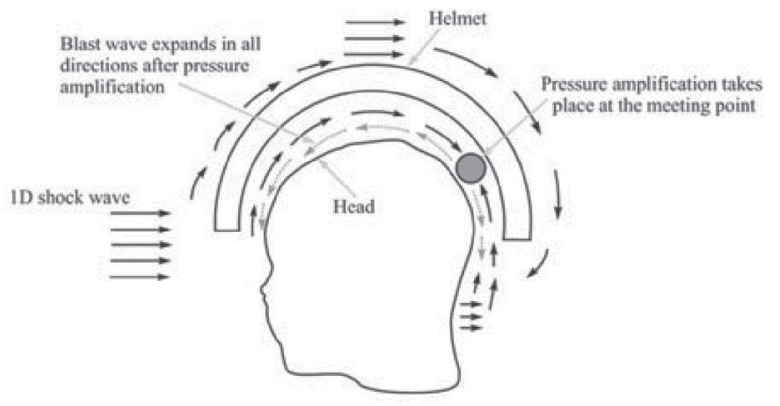

(a)

Pressure (MPa)

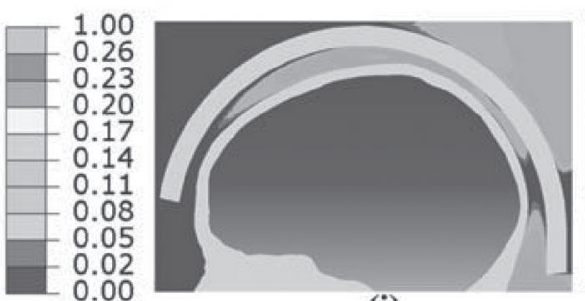

(i)

$\mathrm{t}=1.05 \mathrm{msec}$

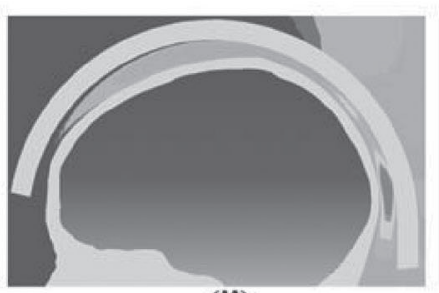

(ii)

$\mathbf{t}=\mathbf{1 . 1 2 5} \mathrm{msec}$

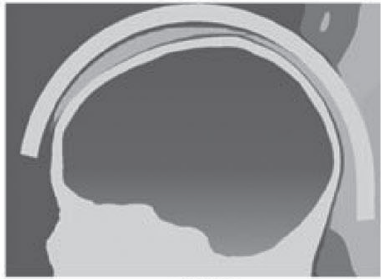

(iii)

(b)

Figure 6. Flow field inside and outside of the head-helmet subspace (3D simulations): (a) schematic explaining underwash effect of the helmet (b) flow fields beneath the helmet.

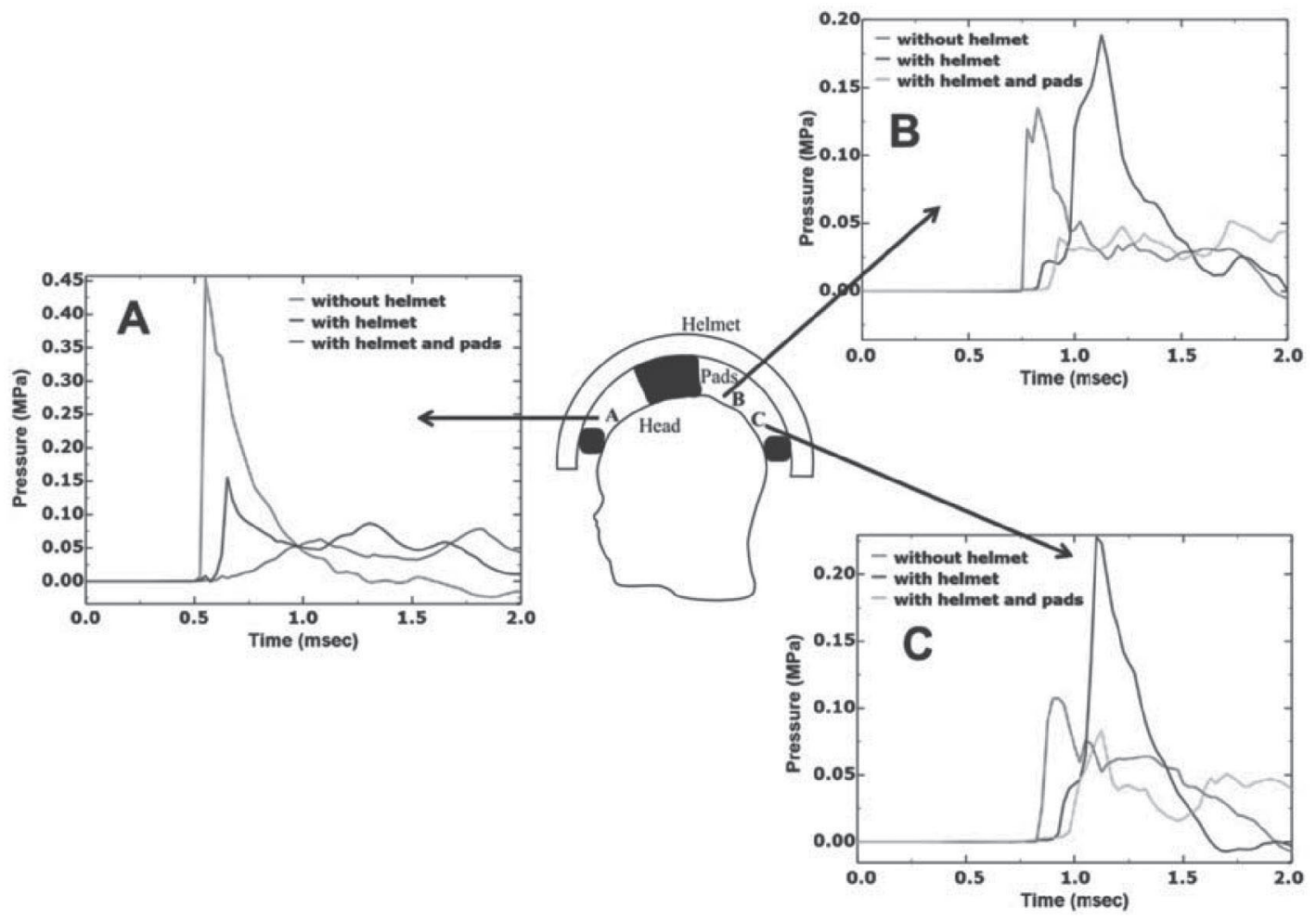

Figure 7. Pressure histories at various locations of head-helmet subspace (midsagittal plane, 3D simulations).

peak pressure is reduced by $65.6 \%$ at location $\mathrm{A}$ (front) and is increased by $39.7 \%$ and $112.5 \%$ at locations B (top) and $\mathrm{C}$ (rear), respectively, with respect to without helmet case (case 1). The increase in peak pressures at locations B and C can be clearly attributed to the underwash effect, discussed previously. 

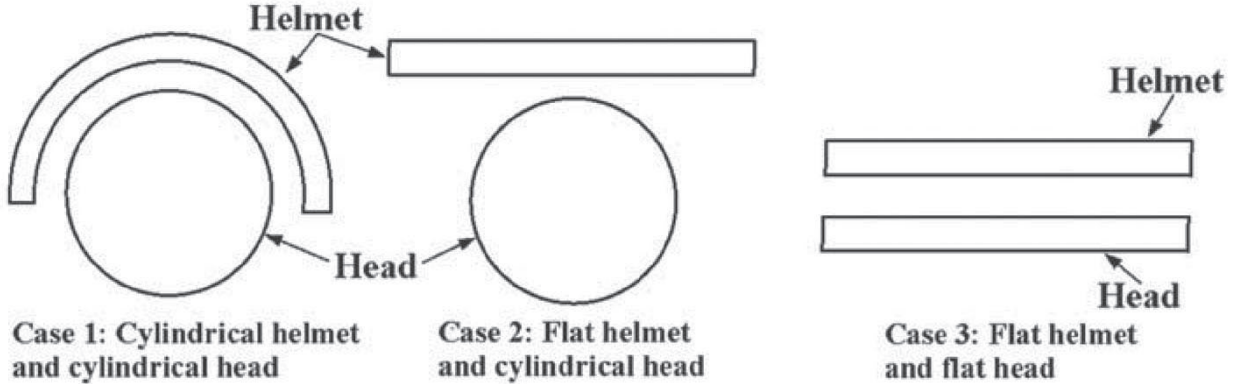

Case 3: Flat helmet and cylindrical head

(a)

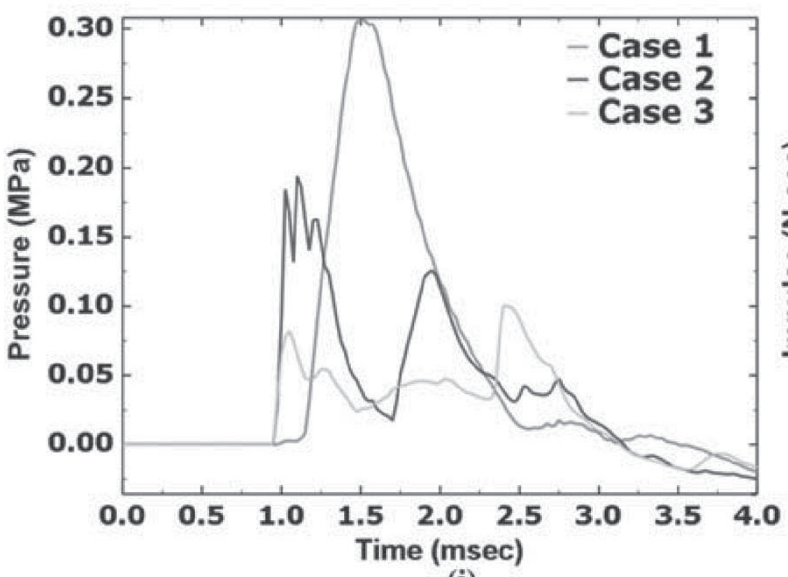

(i)

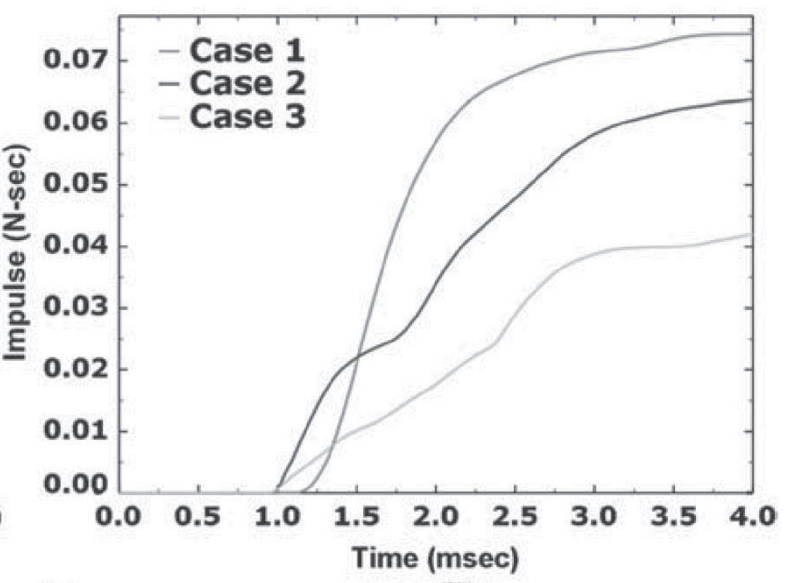

(b)

Figure 8. Effect of curvature of the helmet and the head: (a) modeling set-up for studying curvature effect of the helmet and the head (b) (i) average pressure in the back region of the head-helmet subspace and (ii) total impulse transmitted to the back region of the head. Incident blast intensity $0.52 \mathrm{MPa}$.

This indicates that intensification of pressure (focusing) exists due to underwash effect arising from the differential flow path. To understand how the underwash influences both the local peak pressure and the impulse, it is postulated that the pressure intensification depends on the shape of the helmet (curvature) and the 3D geometry of the headhelmet subspace (gap) with respect to the oncoming pressure wave and its characteristics, e.g. pressure, velocity and rise/fall time. These aspects are studied in the following section. It should be noted that local peak pressures in the head-helmet subspace and impulse transmitted to the head are analyzed as these quantities determine the effective load on the head.

\subsection{Effect of curvature, head-helmet gap size and incident peak pressure intensity}

To examine the effect of geometry, three different cases are considered. In the first case, the head and the helmet are modeled as cylinders; in the second case, the head is cylindrical and the helmet flat and in the third case, both the helmet and the head are flat (Figure 8(a)). In all these cases, there is a constant gap of $13 \mathrm{~mm}$ between the helmet and the head. Figure 8(b-i) and (b-ii) shows the pressure and impulse profiles at the back of the head-helmet sub- space in which the focusing occurs. It is clear from Figure 8(b) that the pressure and impulse are increased when both the shapes are cylindrical in comparison with the other two cases. This trend is the same when the incident overpressure is increased from 0.18 to $0.52 \mathrm{MPa}$.

Having identified that the cylindrical case offers the most severe loading conditions, this case is used to study the effect of head-helmet gap size and incident peak pressure intensity on the underwash. Figure 9 shows the $P_{\max } / P^{*}$ (normalized peak maximum overpressure) in the head helmet subspace as a function of gap size for different incident peak pressure intensities $P^{*}$. As the gap is reduced, pressure in the gap increases $(P \alpha 1 / V, V$-volume). Thus, $P_{\max } / P^{*}$ increases as the gap size is reduced till certain critical gap size. Thereafter, the boundary effects become dominant and $P_{\max } / P^{*}$ decreases due to these boundary effects. It should also be noted that the $P_{\max } / P^{*}$ is increased as incident peak pressure intensity $P^{*}$ is increased. Numerical simulations indicate that for the ranges tested, the angle $\theta$ at which $P_{\max }$ occurs is between $140^{\circ}$ and $155^{\circ}$.

Another quantity of interest is the transmitted impulse, $I$, and depends on the maximum peak pressure, $P_{\max }$ and rate of pressure decay (i.e. rate of expansion) once $P_{\max }$ is established. The higher the $P_{\max }$ and lower the rate of pressure decay, the higher is the impulse transmitted. As shown earlier, the $P_{\max }$ increases as the gap size is 


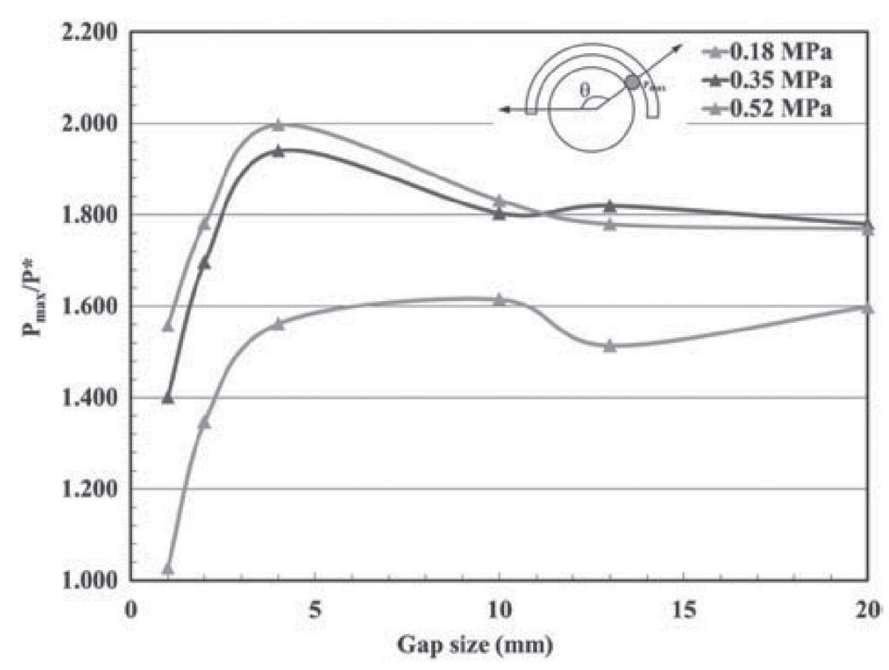

Figure 9. Normalized maximum peak overpressure in the head-helmet subspace $\left(P_{\max } / P^{*}\right)$ as a function of gap size for different incident blast intensities $P^{*}$.

reduced till critical gap size. The rate of pressure decay, however, decreases continuously (no critical gap size) as the gap size is decreased as shown in Figure 10(a). This is due to the fact that as the gap size is reduced, there is not enough space for expansion, and boundary reflection effects become dominant. Similar observations are reported by Rafaels et al. (2010) from their blast experiments on helmeted head. From our simulations, it was found that, for a given incident peak pressure intensity $P^{*}$, rate of pressure decay contributes more to impulse transmitted to the head than $P_{\max }$. Hence, for a given incident peak pressure intensity $P^{*}$, impulse transmitted to the head continuously increases as the gap size is reduced as shown in Figure 10(b).

\subsection{Effect of supporting pads}

Earlier analyses indicate that underwash effect increases both the peak overpressure and transmitted impulse to the rear side of the head when the head is covered with helmet and there is a gap between them. However, our simulations show that the underwash effect is absent with the presence of tightly fitting foam pads that effectively block any flow in the head-helmet subspace (Figure 7). Let us recall that, Figure 7 compares the pressure history at various locations of head-helmet subspace (midsagittal plane) of cases without helmet, with helmet and gap, and with helmet and pads, and that we referred to these three cases as 1,2, and 3, respectively. The peak pressure is reduced by $86.08 \%$ and $59.50 \%$ at location A (front) for case 3, compared with cases 1 and 2, respectively. Similarly, the peak pressure is reduced by $61.86 \%$ and $72.7 \%$ at location B (top), for case 3 compared with case 1 and 2 , respectively. Similar reduction in the peak pressure is seen at location C (rear) at a level of $22.44 \%$ and $63.5 \%$, respectively. In general, it is clear that significant reductions in peak overpressure are experienced when the helmet is worn with pads tightly filling the head-helmet subspace compared with other cases of no helmet or helmet with gap.

One argument against pads is that though the pads reduce the underwash overpressure, they strongly couple helmet-head motion, thus increasing the effective load on the head (Moss et al. 2009). From our simulations, we found that maximum displacement of the head is $6,1.28$, and $2 \mathrm{~mm}$ for cases 1, 2, and 3, respectively. Total impulse transferred to the head is decreased by $40 \%$ for case 3 compared with other two cases. Thus, even though pads strongly couple the helmet motion with the head, total impulse transferred to the head with supporting pads is lower than the other two scenarios.

In our simulations, six pads on the perimeter of the helmet are perfectly resting (initially) on the outer perimeter of the head/skull (ideal case) and the crown pad is slightly offset from the head (due to difference in curvature of the helmet and the head). The actual scenario in the battlefield is somewhere between constant gap between helmet and head and suspension pads perfectly resting (ideal case) on the head. In actual field scenario, the helmet is com-
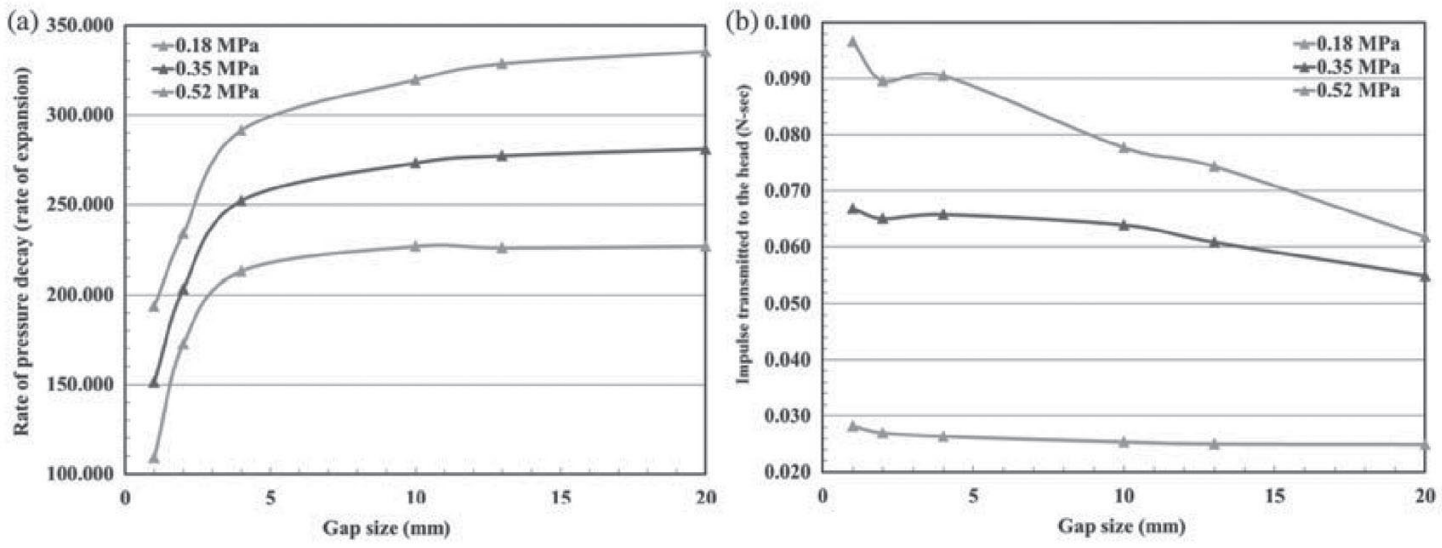

Figure 10. (a) Rate of pressure decay in head-helmet subspace (b) impulse transmitted to the head as a function of gap size for different incident blast intensities $P^{*}$. 


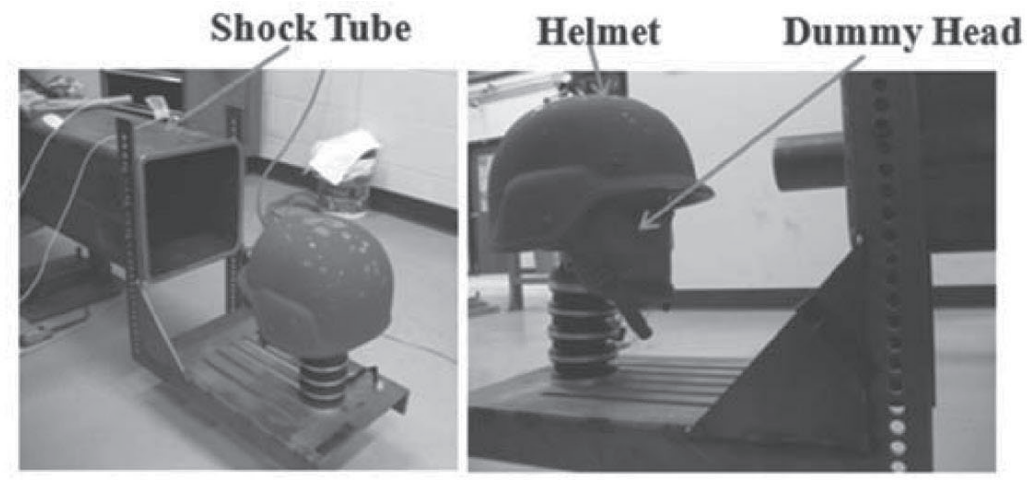

(a)

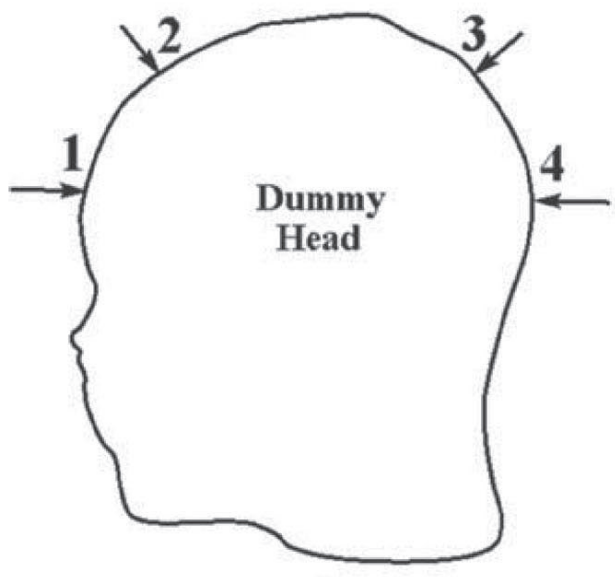

(b)

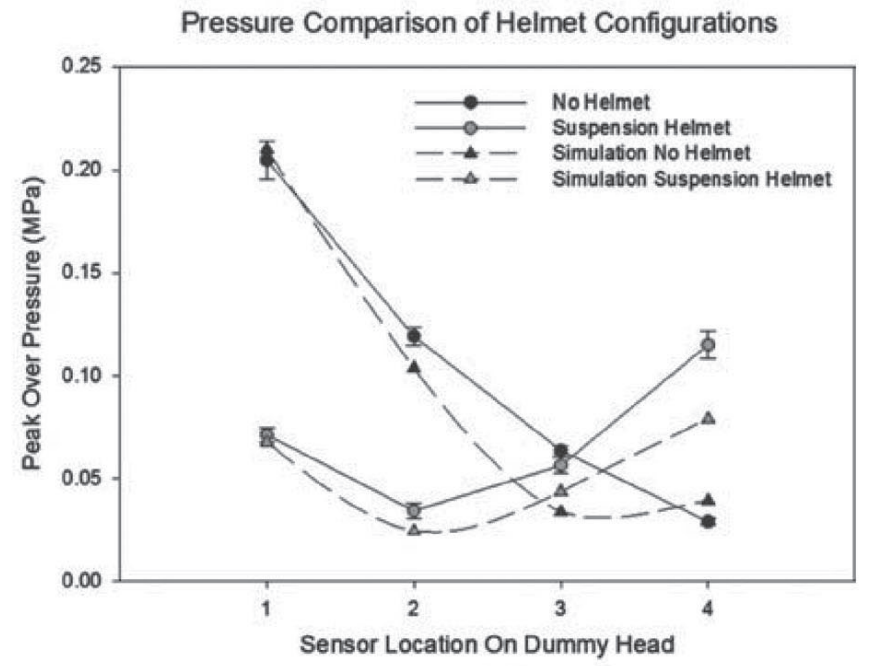

(c)

Figure 11. (a) Experimental set-up (b) sensor locations (c) peak pressures at sensor locations for blast experiments carried out to evaluate performance of the helmet.

fortably located on the head, which may not fit the definition of tightly fitted. In addition, these helmets and pads are designed in three broad sizes namely large, medium, and small. Hence, it is impossible to have perfect fitting of the helmet on the head due to huge variation of head sizes among the battlefield crew. In addition, these pads can disintegrate quickly in the rigors of combat. Thus, our 3D simulation models of cases 2 and 3 present two extreme cases and the actual battlefield condition lies between them. We have conducted a simulation with the pads but with a gap between the pads and the head; underwash was still observed in this case. Thus, parametric studies on head helmet gap size and incident pressure intensity are still important if we were to optimize geometry of the helmet, gap $g$, and the pads, to reduce the peak pressure and total impulse on the head.

\subsection{Experimental validation of underwash effect}

Our simulation results are validated against in-house experiments and published field experimental data (Mott et al. 2008; Rafaels et al. 2010). Experiments were carried out with the dummy head, both with and without helmet, kept $22.2 \mathrm{~cm}$ outside the 9-inch shock tube as shown in Figure 11(a). The sensor locations are marked in Figure 11(b). The experiments are the replication of gap case (case 2) between the head and the helmet. The 3D simulation with the dummy head outside the shock tube was developed to replicate experimental set-up. Figure 11(c) plots peak pressures at each sensor location from experiments and simulations. The simulation results match very well with the experiments both quantitatively and qualitatively. Sensors 3 and 4 indicate higher peak overpressure for "suspension helmet" case than for "no helmet" case, thus confirming "underwash effect" under the helmet. The maximum deviation between experiment and simulation is $47.16 \%$ at sensor 3 for no helmet case. Small quantitative differences between simulation and experiments can be attributed to the fact that suspension inside the helmet was not modeled in the simulations and to the mesh size, which may not match the node value with the precise location of the sensor.

Rafaels et al. (2010) carried out experiments on postmortem human subjects and Hybrid III dummy to understand blast response with and without helmet. From their measurements, they found an increase in peak pressure 
and total impulse for two locations (crown and right ear) that were under the helmet compared with no helmet case. Mott et al. (2008) conducted tests on Hybrid III dummies. All the tests were carried out in three different blast orientations: front, back, and side. From their measurements, they found equivalent or higher pressures under the helmet than no helmet case for the sensors that were away from incident blast location. These results are consistent with the trends predicted from our simulations.

\section{Summary and conclusions}

The role of helmets in mitigating the effect of primary shock waves is not clearly understood. Though the current helmets have not been specifically designed for preventing blast-induced TBIs, understanding the critical issues related to current helmets is important for developing a better helmet against shock blasts. In this work, the primary shock wave interactions for various helmet head configurations were evaluated. The pressure and impulse intensification effects were elucidated as a function of geometry, head-helmet gap, and surface curvature. Though this work primarily focused on a clean Friedlander wave (which is important to establish/understand mechanisms), the basic understanding and the results are valid for complex cases of shock waves encountered in the field.

Some of the key findings of this work are as follows:

- When a shock wave encounters human head, the highest reflected overpressure occurs in the regions of concavity, notably at nasion (the nose-eye cavity). The reflected overpressure increases by $4.61,2.62$, and 2.71 times the incident overpressure at nasion, nosetip, and forehead, respectively, for an incident blast intensity of $0.18 \mathrm{MPa}$.

- Curvature of the helmet and the head governs the flow fields around the head and has great influence on the pressure and loadings experienced by the head.

- When a gap exists between the head and the helmet, there is an increase in overpressure and impulse on the rear side of the head. This increase is a nonlinear function of gap size and incident overpressure. Total impulse on the rear side of the head is increased by $13.13 \%, 21.69 \%$, and $56.39 \%$ for incident blast intensities of $0.18,0.35$, and $0.52 \mathrm{MPa}$, respectively, as gap size is reduced from 20 to $2 \mathrm{~mm}$. The location of maximum overpressure $\left(P_{\max }\right)$ is observed at about $140^{\circ}$ from the direction of the oncoming wave.

- Though there is an increase in overpressure and impulse on the rear side of the head when a gap exists between the head and the helmet, these overpressure and impulse are still lower than the absolute values encountered in the front side (oncoming blast wave side) of unprotected head. The average peak overpressure and total impulse on the front side of the head without the helmet are $0.82 \mathrm{MPa}$ and $0.1031 \mathrm{Ns,}$ respectively, for incident blast intensity of $0.52 \mathrm{MPa}$.
The average peak overpressure and total impulse on the rear side of the head with the helmet are 0.30 $\mathrm{MPa}$ and $0.0743 \mathrm{Ns}$, respectively, for incident blast intensity of $0.52 \mathrm{MPa}$. A similar trend is observed at lower intensities. This indicates that the helmet is serving a certain degree of protection against shock. However, a better design is desired to reduce the underwash.

- Tight foam pads between the head and the helmet eliminate the impulse and overpressure increases. This case offers best protection, preventing any pressurization in the head-helmet subspace (gap). The maximum reduction in the overpressure and impulse with the pads are observed to be $86.08 \%$ and $20.15 \%$, respectively.

- The underwash effect is confirmed by the shock tube experiments. Simulations and experiments are in good agreement.

Acknowledgments - The authors acknowledge the financial support provided by the US Army Research Office for the project on "Army-UNL Center for Trauma Mechanics," Contract No. W911NF-08-1-0483. The authors thank Ruqiang Feng, Mehrdad Negahban, and Erwan Plougonven at UNL for many useful discussions. The authors also thank David Mott of Naval Research Laboratory and Robert Salzar of University of Virginia for bringing their experimental work to our attention.

\section{References}

Aare M, Kleiven S. 2007. Evaluation of head response to ballistic helmet impacts using the finite element method. Int J Impact Eng. 34:596-608.

Anderson J. 2001. Fundamentals of aerodynamics. New York: McGraw-Hill.

Baker WE. 1973. Explosions in air. Austin, TX: University of Texas Press.

Bhattacharjee Y. 2008. Shell shock revisited: solving the puzzle of blast trauma. Science. 319:406-408.

Bowen LG, Fletcher ER, Richmond DR. 1968. Estimate of man's tolerance to the direct effects of air blast. Technical Report, DASA-2113, Defense Atomic Support Agency, Department of Defense, Washington, DC.

Cernak I, Wang Z, Jiang J, Bian X, Savic J. 2001. Ultrastructural and functional characteristics of blast injury-induced neurotrauma. J Trauma. 50(4):695-706.

Chafi MS, Karami G, Ziejewski M. 2010. Biomechanical assessment of brain dynamic responses due to blast pressure waves. Ann Biomed Eng. 38(2):490-504.

Chen Y, Ostoja-Starzewski M. 2010. MRI-based finite element modeling of head trauma: Spherically focusing shear waves. Acta Mechanica. 213(1-2):155-167.

Courtney AC, Courtney MW. 2009. A thoracic mechanism of mild traumatic brain injury due to blast pressure waves. Med Hypotheses. 72(1):76-83. 
Courtney AC, Courtney MW. 2011. Working toward exposure thresholds for blast-induced traumatic brain injury: thoracic and acceleration mechanisms. NeuroImage. 54 (Suppl. 1): S55-S61.

Ganpule S, Gu L, Chandra N. 2010. The effect of shock wave on a human head. Proc ASME Int Mech Eng Congress Exposition (IMECE). 2:339-346.

Lew HL, Poole JH, Alvarez S, Moore W. 2005. Soldiers with occult traumatic brain injury. Am J Phys Med Rehab. 84:393-398.

Ling G, Bandak F, Armonda R, Grant G, Ecklund J. 2009. Explosive blast neurotrauma. J Neurotrauma. 26(6): 815-825.

Moore DF, Jerusalem A, Nyein M, Noles L, Jaffee MS, Radovitzky RA. 2009. Computational biology: Modeling of primary blast effects on the central nervous system. NeuroImage. 47:T10-T20.

Moss WC, King MJ, Blackman EG. 2009. Skull flexure from blast waves: A mechanism for brain injury with implications for helmet design. Phys Rev Lett. 103:10.

Mott DR, Schwer DA, Young TR, Levine J, Dionne JP, Makris A, Hubler G. 2008. Blast-induced pressure fields beneath a military helmet. Proceedings of the 20th international symposium on military aspects of blast and shock; September 1st-5th, Oslo, Norway.

Okie S. 2005. Traumatic brain injury in the war zone. N Engl J Med. 352:2043-2047.

Rafaels KA, Shridharani J, Bass CR, Salzar RS, Walilko TJ, Panzer MB. 2010. Blast wave attenuation: Ballistic protective helmets and the head. Washington, DC: Personal Armor Safety Symposium (PASS).

Reynosa MA. 1999. The Personnel Armor System Ground Troops (PASGT) helmet. Atglen, PA: Schiffer Military History.

Taylor PA, Ford CC. 2009. Simulation of blast-induced earlytime intracranial wave physics leading to traumatic brain injury. J Biomech Eng. 131:6.

Xydakis MS, Bebarta VS, Harrison CD. 2007. Tympanic membrane perforation as a marker of concussive brain injury in Iraq. N Engl J Med. 357(8):830-831.

Zhang L, Yang KH, King AI. 2001. Comparison of brain responses between frontal and lateral impacts by finite element modeling. J Neurotrauma. 18(1):21-30. 